\title{
El desarrollo endógeno y aprendizaje institucional: Una relación recursiva
}

\author{
Petrizzo Páez, María Angela*
}

\section{Resumen}

"Este artículo ilustra el modo en que el aprendizaje en las instituciones públicas puede operar como catalizador de la formación ciudadana y como operador fundamental en el proceso de Desarrollo Endógeno, configurándose una relación recursiva entre ambos. En este sentido y partiendo de una propuesta cercana a las ciencias gerenciales acerca del aprendizaje organizacional, se asume al aprendizaje institucional como su equivalente a la luz de lo que Boisier (2002) llama conversaciones sociales, y el modo en que éstas contribuyen por una parte a la generación de sinergias cognitivas entre sus participantes y, por la otra, a la construcción de un discurso de la acción política más apropiado tanto para las instituciones públicas como para los ciudadanos. Los procesos de aprendizaje institucional pueden ser observados a través del análisis del discurso de las instituciones públicas y de la posibilidad construir internamente una memoria colectiva. De la propuesta presentada, se desprende la posibilidad de observar si, a través del ejercicio de las funciones básicas de las instituciones públicas esto es, custodiar y gestionar lo público, la presencia de un proceso de aprendizaje colectivo y crítico revertido hacia los ciudadanos y que cuente con su participación".

Palabras clave: Desarrollo Endógeno y ciudadanía, aprendizaje institucional.

\section{Endogenous Development and Institutional Learning: a Recursive Relationship}

\section{Abstract}

"This article purports to illustrate how learning in public institutions can operate as a catalyst for civic education and as a key operator in the endogenous development process, shaping a recursive relationship between them. In this regard, and based on a proposal about organizational learning that is close to management sciences, institutional learning is assumed to be its equivalent in the light of what Boisier (2002) calls "social conversations" and how these contribute on the one hand to the generation of cognitive synergies among their participants and, on the other, to the construction of a discourse for political action that is more appropriate for public institutions as well as for citizens. Organizational learning processes can be observed through analyzing the discourse of public institutions and the possibility for constructing a collective memory internally. From the proposal presented, the

Recibido: 27-02-07. Aceptado: 16-10-07

* Politólogo. Analista de Proyectos de la Unidad de Gestión en Ciencia y Tecnología de FUNDACITE, Mérida. E-mail: petrizzo@gmail.com 
possibility arises for observing whether, by exercising the basic functions of public institutions, that is, preserving and managing what is public, the presence of a collective and critical learning process can be seen that reverts toward the citizens and counts on their participation".

Key words: Endogenous Development and Citizenship/Institutional Learning.

\section{Introducción}

El carácter endógeno en la construcción de un desarrollo que se inicie desde la definición del bien común frente a lo público y que incentive la activación de fuerzas sociales locales de modo que tributen a esa construcción, demanda de las instituciones públicas la doble tarea de liderar procesos de aprendizaje tanto en su estructura como hacia los ciudadanos, a fin de propiciar en estos últimos un cambio de actitud que permita desplegar su quehacer de un modo armónico.

Este artículo parte de la premisa que la actividad primigenia de todo ciudadano es la búsqueda de su despliegue como ser humano hacia su espacio inmediato: la comunidad, la ciudad. Se asume, por tanto, que el ejercicio de la ciudadanía pasa por la acción humana sobre ese entorno y sobre todos los otros actores que lo conforman. Además se propone que ello genera no sólo un ejercicio responsable de la condición de ciudadano, sino también la construcción del arraigo entre la persona y el entorno con el que interactúa. En otras palabras, la condición de ser humano, en interacción con un entorno, hace de la persona un ciudadano y cuando éste se siente parte fundamental del destino común de su entorno, cuando siente y construye su arraigo social y cultural con la comunidad de la que forma parte, avanza en conjunto hacia el desarrollo endógeno, en un proceso en el que, al mismo tiempo, se forma en el ejercicio de su ciudadanía.

En Venezuela, tras 48 años de democracia, en algunos espacios del quehacer social se hace evidente el deterioro de los vasos comunicantes entre instituciones y ciudadanos. Por una parte, vemos a ciudadanos desarraigados que regresan a su condición previa de individuos y que no albergan el deseo de apropiarse de los espacios públicos de decisión política que las democracias ofrecen por naturaleza. $Y$, además, privilegian la obtención de beneficios propios por encima del rescate del valor de lo público y común a todos. También se observa a instituciones que no muestran su compromiso con la labor de construcción del desarrollo desde el espacio en el que la acción de esa institución se despliega en el ámbito comunitario (Petrizzo, 2005). Ambos, ciudadanos e instituciones, están llamados a encontrarse en el espacio de la construcción del bien público, a conversar, a dialogar, de un modo en que se posibilite la construcción de escenarios viables para el Desarrollo Endógeno.

La construcción del diálogo necesario entre instituciones y ciudadanos, partiendo de aquello que Boisier (2002) llama conversaciones sociales, apunta hacia la materialización de un compromiso con el camino del Desarrollo Endógeno y, además, abre la posibilidad de iniciar un proceso de generación y formación de un ciudadano activo y responsa- 
El desarrollo endógeno y aprendizaje institucional: Una relación recursiva Petrizzo Páez, María Angela

ble con la construcción de la sociedad e instituciones con las que interactúa.

La idea de Desarrollo Endógeno que aquí se plantea se distingue de la tradicional noción de desarrollo en tanto que progreso, y se acerca mucho más a una noción social y humanística del término ${ }^{1}$. No se trata, por tanto del ajuste necesario de los planes y programas gubernamentales en la búsqueda de logro de estándares de progreso preestablecidos y acordes con otras realidades sociales y políticas, si no la búsqueda de las respuestas propias en torno a preguntas sobre cómo redimensionar las relaciones del ser humano con su entorno para hacer más viable su coexistencia y su despliegue integral.

Aquí se pretende, por tanto, abonar el terreno en la exploración de la pertinencia dentro de cualquier proyecto de Desarrollo Endógeno, de que tanto las instituciones públicas y ciudadanos posibiliten, gracias a su diálogo, la revisión de nuevos modos de vincularse tributando, en conjunto y no ya de forma desarticulada, a esa construcción común de un destino también común cifrado en la búsqueda del bien público. Adicionalmente, el presente artículo pretende exponer ante el lector los espacios que el aprendizaje institucional debiera apuntalar en un proceso de construcción del Desarrollo Endógeno, y el modo en que ese aprendizaje podría ser observable.

\section{Las instituciones públicas como custodias del aprendizaje colectivo}

Aprender es sinónimo de una progresiva adquisición de conocimiento que, de algún modo, conduce a modificar hábitos y conductas, lo cual puede observarse gracias a la interacción de quien aprende con su entorno. Haciendo una extensión de esta definición podríamos decir que el aprendizaje colectivo, en comunidad, conduce de modo directo a una formación ciudadana $y$, desde allí, a la reconstitución del sentido común de la revalorización del papel del ciudadano en la constitución y preservación del bien público. Si asumimos al Desarrollo Endógeno como aquello que activa fuerzas sociales en torno a la construcción y preservación de lo público, diremos entonces que el aprendizaje colectivo es la puerta hacia el carácter endógeno del desarrollo.

Dicho lo anterior, es importante resaltar que el aprendizaje responde a una doble esfera en la que obra el ser humano: la del individuo y la del colectivo. En tanto que el individuo aprende y, al mismo tiempo, acciona en función del aprendizaje adquirido y que, también, el colectivo aprende, se adapta y se reconstruye en función del aprendizaje que se opera primero en el individuo, incidiendo nuevamente en la esfera de cada cual. Lejos de ser un estadio o punto acabado, el apren-

1 Desarrollo como "despliegue", es decir, desdoblaje de aquello que está plegado sobre sí mismo. 
dizaje es un proceso en el que se obra, en última instancia, el cambio de actitud(es) en el sujeto que aprende ${ }^{2}$. De este modo, debe partirse del acuerdo que el despliegue del ser humano no puede entenderse sin la observación del proceso de aprendizaje que se inicia aún antes del surgimiento de ambos (individuo y aprendizaje) y que en este proceso, los cambios en el entorno, dada su complejidad y duración en el tiempo, observan una lentitud mayor (Brown, Collins, Duguid, 1989; y Wilson y Cole, 1991).

Esto supone que el aprendizaje es posible gracias a la construcción y preservación de una suerte de memoria común. Una sociedad sin memoria, niega la generación de conocimiento en su seno y niega la posibilidad de que sus instituciones fortalezcan procesos de formación ciudadana. De modo que aquello que se dijo antes que es la puerta hacia el Desarrollo Endógeno sólo puede alcanzarse con la memoria colectiva como llave. Y allí, ésta última funciona, además, como elemento de anclaje, de vínculo ineludible del ciudadano con su entorno. Se erige, entonces, como un eslabón del arraigo del ciudadano con su comunidad, superando así una concepción de arraigo del individuo que supone la mera localización geográfica en un espacio determinado. Arraigo es, en términos del Desarrollo Endógeno, el resultado de la conjunción de diversos elementos que desde el quehacer diario de una comunidad contribuyen a que cada ciudadano se sienta y viva como parte integral de ésta y responsable de su suerte. De allí que la memoria colectiva sea un eslabón del arraigo del ciudadano, además de pilar del aprendizaje, y clave de la construcción de un Desarrollo Endógeno que, como se verá después, sea percibido en tanto sean observables sus dimensiones (decisión, control y enriquecimiento locales). Pero sobre ello se volverá más adelante.

Como proceso, la noción de aprendizaje encierra también la idea de voluntad, concebida como una motivación favorable hacia una acción o cosa, más allá del contenido que ésta encierre ${ }^{3}$. En el caso que nos ocupa, debe decirse que el aprendizaje encierra la voluntad positiva hacia emprender el camino del cambio individual y colectivo. Este cambio, se inicia con el individuo y, desde allí, recordando las dos esferas en que opera el aprendizaje, involucra también a las organizaciones de las que el individuo puede formar parte o con las que se vincula de algún modo. Dada la complejidad manifiesta de las sociedades modernas, resulta obvio, por tanto, que el proceso de aprendizaje no es unidireccional, lineal, ni unidimensional. Allí la complejidad del aprendizaje radica en el carácter multi-dimensional del desarrollo mismo del ser humano en

2 Sea este un actor colectivo o individual.

3 En otras palabras, la "positividad" de la voluntad no radica en que lo que se desea hacer sea bueno per se, sino en el hecho que el individuo o el colectivo tenga la intención cierta de emprender el camino hacia esa cosa que se desea hacer. 
El desarrollo endógeno y aprendizaje institucional: Una relación recursiva Petrizzo Páez, María Angela

su construcción de la sociedad, lo que permite ver matices entre individuos, organizaciones o colectividades.

Este proceso continuo de adquisición de conocimiento y motivación positiva hacia el cambio ocurre gracias, tanto a la acción de ámbitos formales (sistema educativo), como de ámbitos informales (familia, amigos, medios de comunicación). Su complejidad es enorme, en vista de que en él intervienen un conjunto de relaciones y vínculos sin que pueda observarse en ellos un inicio y fin de forma clara.

Retomando la premisa inicialmente expuesta acerca del aprendizaje como puerta hacia el Desarrollo Endógeno, en un marco en el que el aprendizaje en colectivo es el garante de la reconstitución del bien público, es importante insistir que el aprendizaje colectivo que se indica como necesario no ocurre en un instituciones autárquicas, sino precisamente desde instituciones públicas que se esfuerzan por aprender y motorizar el aprendizaje ciudadano.

\section{El aprendizaje en instituciones públicas}

Asumiendo que buena parte del aprendizaje individual acaece en el marco de organizaciones, estén o no orientadas hacia la canalización de procesos educativos y que éstas son en muchos casos formales, dado que también estas organizaciones son receptoras del aprendizaje individual, pues, como hemos visto, se trata de un proceso complejo y bidireccional y sabido, además, el peso que las organizaciones sociales tienen en la adaptación y alineación de la conducta individual; resulta clave detenerse en lo que significa el proceso de aprendizaje enmarcado en un contexto organizacional público.

El desarrollo de la noción de aprendizaje en las instituciones, en adelante aprendizaje institucional, coincide con la búsqueda de un remozamiento en las teorías organizacionales tradicionales en el plano de la gerencia de organizaciones. En este sentido, el aprendizaje institucional se concibe como un proceso de detección y enmienda de errores a la luz del desempeño de una organización (Argirys, 1978). Esta definición es complementada por McGill (1994), enfatizando que en buena medida este proceso ocure de forma voluntaria, pues le permite a la organización ganar comprensión con respecto a su entorno y adaptarse; es decir, generar conocimiento, construir memoria y desde allí, aprender.

Entender el aprendizaje institucional, tal y como ocurre con el aprendizaje individual, puede implicar aproximarse a él como un proceso de cambio, como un proceso simple de adquisición de conocimiento o como un proceso integral donde el aprendizaje es catalizador del cambio a través de la adquisición de conocimiento pertinente y válido sobre el entorno y sobre la organización misma.

El aprendizaje institucional como cambio está vinculado a la capacidad de las organizaciones para operar cambios en su interior. En este sentido, el aprendizaje institucional ocurre en función del entorno de la organización y allí puede operar como un instrumento que previene o prepara hacia el entorno (visión proactiva) o como una herramienta que facilita acoplamiento de la organización a este (visión adaptativa). Según como muestra Nekane (2000), durante los años 
60 y 70 del siglo $X X$, se observa una preeminencia de enfoque de adaptación y, a partir de los años 80 , se observa una exacerbación del enfoque proactivo. Interesa aquí, resaltar la idea del aprendizaje de las organizaciones como resultado de la interacción de éstas con su entorno (Cyert y March, 1963; Cangelosi y Hill, 1965; Duncan, 1974; March y Olsen, 1976; Duncan y Weiss, 1979 y Hedberg, 1981). Esta línea constructivista plantea para la organización un análisis de las condiciones del entorno que se revierte en un aprendizaje en función de la experiencia y en la corrección de errores que posibilita que la organización se adecue al entorno en el cual ella despliega sus acciones.

Viendo al aprendizaje institucional desde la óptica de un proceso de adquisición y gestión de conocimiento $\mathrm{Hu}$ ber, 1991; Quinn, 1992; Revilla, 1996; Nonaka y Johansson, 1985; Nonaka, 1988 y 1991; Nonaka y Takeuchi, 1995; Nonaka e Ichijo, 1997 y Nonaka, Reinmoellery Senoo, 1998), se le observa signado por los modos en que las organizaciones potencian, crean, generan, organizan y difunden nuevos conocimientos y rutinas de actividades, dentro de sus propias culturas o climas organizacionales. En estos términos, el conocimiento contribuye, en sí mismo, a la ampliación de la base sobre la cual se sustenta su generación, de forma tal que posibilita el fortale- cimiento de las propias capacidades de las organizaciones y las prepara en el marco de la resolución de sus problemas. Moreno y Rodríguez (2002) refieren en el trabajo de Heidi Amponsem una ampliación de esta idea cuando muestra una suerte de asimilación del conocimiento individual por parte de la organización, de forma que parte de ese proceso de generación y enriquecimiento del conocimiento organizacional está estrechamente vinculado con el conocimiento que el individuo ha asimilado en tanto que tal.

Finalmente, el aprendizaje institucional puede entenderse también como una combinación de un proceso generador o potenciador de cambios y vinculado estrechamente a la idea de adquisición y gestión del conocimiento. Es en este marco en donde surge el concepto de la organización que aprende o la llamada por Senge (1990), organización inteligente.

Es importante hacer un apartado sobre el tema del conocimiento en las organizaciones o instituciones. Puntualizar qué es conocimiento y qué no en una organización pública puede ayudar a discernir sobre el modo en que ese conocimiento permite la generación de memoria colectiva y, por tanto, de aprendizaje institucional. Nonaka y Takeuchi (1994) mostraron un modelo para ilustrar la generación de conocimiento. En la base de este modelo se encuentra tanto el conocimiento explícito ${ }^{4}$, como el tácito ${ }^{5}$. Y según

4 Externalizable, cuantificable, y con gran facilidad de transmisión a través de datos, información sobre resultados o procedimientos u otro mecanismo.

5 Con origen en la experiencia individual de características más íntimas, personales, algunos ejemplos son la intuición, los ideales, los valores. Son difíciles de ser externalizados. 
El desarrollo endógeno y aprendizaje institucional: Una relación recursiva Petrizzo Páez, María Angela

este modelo ${ }^{6}$ (Diagrama 1) la generación, apropiación y transformación del conocimiento se realiza a través de un proceso no lineal que los autores identifican con la forma de una espiral, en donde el inicio es el conocimiento tácito del individuo, convirtiéndolo en explícito a través del proceso de socialización del mismo. El conocimiento explícito, una vez socializado, se combina e interioriza para convertirse, nuevamente, en tácito y obrar como guía de las acciones individuales u organizacionales. Lo interesante de este modelo es la forma en que se muestra que el individuo y su conocimiento obran sobre los de la organización y viceversa.

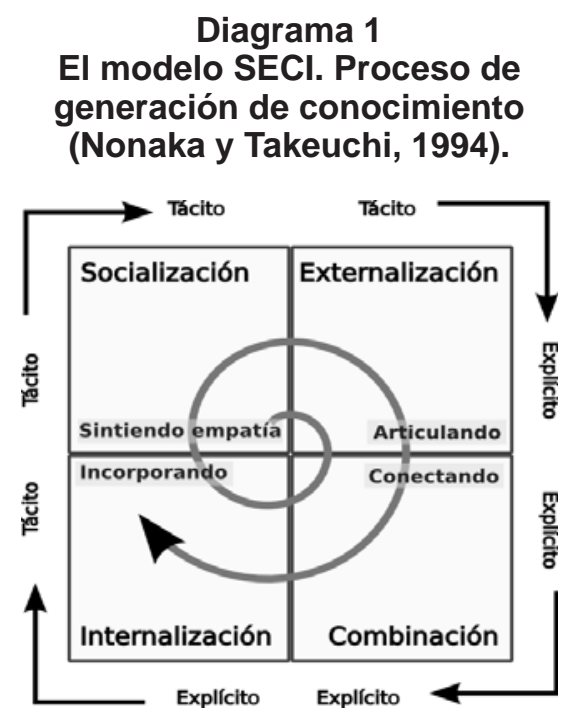

Fuente: http://www.12manage.com/methods_nona ka_seci_es.html (Traducción propia).
El modelo SECl se construye y sostiene a través de la activación del $B a^{7}$, que es el contexto o espacio compartido en el que el conocimiento se origina y se intercambia a través de la interacción de los individuos con las organizaciones y viceversa. Parece que los autores identifican el $\mathrm{Ba}$, como sólo posible de ocurrir en el marco de una organización y de una forma similar a la que se ilustra en el Diagrama 2.

El Ba se origina, por ejemplo a partir del intercambio entre los miembros de una organización (conversación entre pares, por ejemplo), una vez que se origina, el $\mathrm{Ba}$ dialoga con el conocimiento tácito de otros y permite, así, construir visiones sobre, por ejemplo, los procesos dentro

\section{Diagrama 2 \\ Tipos de $B a$, y contextos en los que se produce (Nonaka y Takeuchi, 1994).}

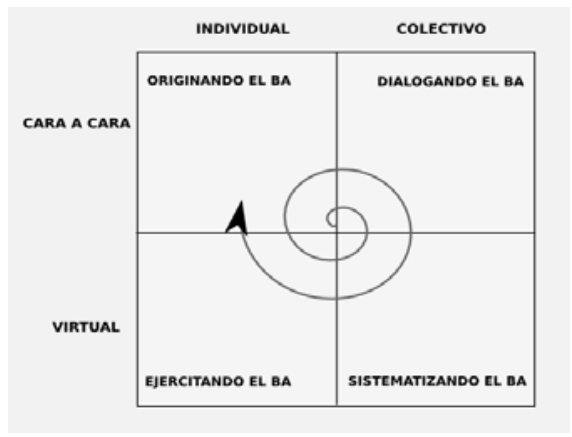

Fuente: http://www.12manage.com/methods_nona ka_seci_es.html (Traducción propia). 
de la organización, haciéndose conocimiento colectivo. A partir de este diálogo, el $B a$ se sistematiza (transformando los datos y visiones e información, por ejemplo) y se hace virtual, para luego aplicarse, contrastando las visiones formadas a través del diálogo, por ejemplo, y enriquecerse, nuevamente, a través del intercambio entre los miembros de la organización.

La esencia del Ba dentro de una institución es bastante similar a aquello que Boisier ha identificado como conversaciones sociales, entendidas como un proceso de construcción y socialización de significados, que ocurre en la relación entre ciudadanos e instituciones (Boissier, 2002). Pese a la cercanía entre ambas ideas, el espacio en que ocurren es algo que las diferencia notablemente. Desde una perspectiva gerencial, los diálogos que favorecen el $\mathrm{Ba}$ ocurren en las organizaciones privadas entre sus miembros o entre éstos y sus clientes. En el ámbito de las instituciones públicas, los diálogos que favorecerían el $B a$ se potencian a través de las conversaciones sociales que ocurren en una comunidad, posibilitando que ciudadanos e instituciones avancen hacia una construcción común basada en el desarrollo de las sinergías cognitivas y potenciadas a través de un proceso inmerso en un complejo entramado de redes inter-ciudadanas e interorganizacionales.
Las sinergías cognitivas, son entendidas por Boisier (2002) como una suerte de relación biunívoca entre los actores vinculados a un tema o ámbito social en particular, que les mueve a accionar de modo conjunto construyendo, por ejemplo, proyectos de acción común. Las sinergías cognitivas se derivan de las conversaciones sociales y están asociadas a la construcción de significados.

Además, hay otro elemento que distingue ambas ideas y tiene que ver con el peso de los conocimientos individuales en la generación del $\mathrm{Ba}$. Mientras para los autores del modelo SECl, el conocimiento individual, que los autores llaman tácito, es entendible en tanto y en cuanto que es beneficioso para la organización; en la noción de conversaciones sociales el conocimiento tácito se involucra de modo activo en las relaciones entre ciudadanos e instituciones de las que ya se habló antes.

En el entorno de las organizaciones, el proceso de la espiral del conocimiento descrito por Nonaka y Takeuchi, permite avanzar hacia lo que Senge (1990) define como el deber ser del aprendizaje en la organización. Lo que este autor denomina organización inteligente es un espacio en el cual es inevitable para el individuo el constante aprendizaje, pues es propicio para alcanzar la realización personal y expandir sus patrones de pensamiento, posibilitando el aprendizaje colectivo. Es un espacio con un $\mathrm{Ba}$ en constante construcción.

$7 \quad$ El término $B a$, carece de traducción al español, al menos una traducción asociada con una única palabra, como sucede con buena parte de los vocablos asiáticos que son más un significado de vida que un contenido semántico lingüísticamente hablando. Sin embargo, diremos que el Ba será el espacio, en donde la generación, intercambio y difusión de conocimiento ocurre. 
El desarrollo endógeno y aprendizaje institucional: Una relación recursiva Petrizzo Páez, María Angela

Para Senge, el aprendizaje es el modo en el que la organización puede lograr los resultados que espera. Bajo esta premisa, la inteligencia es la capacidad de aprendizaje, de allí que pueda decirse que la inteligencia conduce $a$ individuos y organizaciones a perseguir el logro de los resultados deseados, buscando para ello las herramientas de las que se dispone utilizar a tal efecto. De esta forma, en el marco de una organización, el individuo está llamado a desarrollar su inteligencia en tanto que es el camino que posibilita el logro de los fines organizacionales. Quedan fuera de este discurso, y de modo deliberado, los resultados que los individuos desean para sí mismos. Esto ocurre porque en la propuesta de Senge para el desarrollo de organizaciones inteligentes repercute de forma directa en la construcción de la sociedad en tanto que son éstas y no los individuos quienes tienen tal responsabilidad.

Para Senge, la organización inteligente, como concepto, se logra a través del desarrollo de cinco disciplinas en el marco de la gerencia y funcionamiento de la organización. Estas cinco disciplinas son:

1) Dominio personal. Esta disciplina sólo es posible con base en el conocimiento de cada individuo sobre sí mismo. A partir del reconocimiento de sus potencialidades, capacidades y limitaciones puede operarse un aprendizaje que conduzca a un cambio de actitud personal gracias al cual le individuo entenderá que su realización personal pasa por la de la organización.

2) Modelos mentales. Esta disciplina conduce al conocimiento de los paradigmas inconscientes que, en cierto modo, pueden contribuir a restringir la visión del mundo de cada individuo. El desarrollo de esta disciplina se traduce en la utilización de modelos mentales para la promoción de formas de comunicación en la organización.

3) Construcción de una visión compartida. Lo cual se alcanza partiendo de la comprensión por parte de cada individuo del modo en que su visión personal puede, además de enriquecer su concepción de vida y desarrollo personales, no apartarse de la visión de la organización. De este modo, una organización debe animar a sus miembros a que construyan una visión personal que de sentido a sus vidas y que posibilite el logro de la visión de la organización.

4) Aprendizaje en equipo. Para el autor el desarrollo de las capacidades de diálogo internamente en los equipos de trabajo y entre éstos son la clave del surgimiento de nuevas y mejores ideas para el beneficio de toda la organización, fortaleciendo el desarrollo de la inteligencia en grupo.

5) Pensamiento sistémico. Este tipo de pensamiento permite que pueda tenerse tanto de la organización como del entorno, en general, una perspectiva integral, entendiendo que los problemas u obstáculos que se presentan no ocurren de forma aislada. Permite la visualización de cuáles acciones deben emprenderse para superarlos, basado en un conocimiento del entorno y de la organización como un sistema.

Del manejo y destreza en el dominio de estas disciplinas se desprende el modo en que la organización se concibe a 
sí misma en el marco de un proceso de aprendizaje. Senge afirma que de estas cinco disciplinas, la quinta constituye el Pensamiento Sistémico, siendo la que le garantiza a la organización el poder potenciar sus propias capacidades de aprendizaje, recordando que tiene que ver con la capacidad de producir los resultados deseados. En este contexto, sociedad y conjunto de organizaciones, sin lugar para la recreación de lo público, son equivalentes. De esta suerte, lo privado lo es todo y no hay lugar para lo público fuera de las organizaciones.

La idea que encierra la propuesta de Senge cuando muestra la disciplina que se refiere al dominio personal parece capturar la noción de lo público desde el terreno de lo colectivo, de lo común a todos, hasta el terreno de lo privado. De esta suerte, la existencia de lo público se hace innecesario, pues toda la realización del individuo, como se ha dicho antes, está en el terreno de lo privado, de la organización. Esta negación de lo público o, mejor, esta suerte de privatización de lo público, lleva la noción de ciudadano constructor de su entorno, en nuestro caso de un entorno entendido en el piso del Desarrollo Endógeno a su mínima expresión y atenta directamente contra el establecimiento y aprendizaje de un patrón de conducta en donde el conocimiento de las potencialidades y capacidades personales aporte luces sobre el rescate y la construcción del bien público.

Lo rescatable de la propuesta de Senge, para el contexto de organizaciones públicas, es pensar en organizaciones cuya inteligencia intrínseca se en- cuentra vinculada con su capacidad de incentivar el aprendizaje, pues constituye una idea potente al dar cuenta de esa inteligencia y aprendizaje en organizaciones públicas, cuyo referente es el diálogo con los ciudadanos en la construcción de lo público. De allí que, la construcción de organizaciones públicas inteligentes y responsables con la constitución de una idea de bien público en la que se inserten los ciudadanos que dialogan con ellas, exige desmontar la idea de felicidad y realización personales que Senge plantea. Precisa rescatar lo público del terreno de lo privado y ponerlo al servicio de la construcción del bien público.

Lograr que las organizaciones públicas se comporten como lo que Senge afirma deben ser organizaciones inteligentes, pasa por redefinir el sentido y contenido de esas cinco disciplinas cuyo desarrollo garantiza la plena conformación de organizaciones inteligentes. Adaptando la idea de Senge sobre el aprendizaje institucional, podríamos decir que las cinco disciplinas deben ser repensadas en términos de un proyecto de sociedad del que puedan dar cuenta, a saber:

1) Dominio personal. En una sociedad en donde se valora y se construye el espacio de lo público, el dominio personal pasa por el compromiso del ciudadano con su entorno y con el enriquecimiento de las relaciones sociales. El ejercicio de una ciudadanía activa que supere la mera concepción legalista del término. En este contexto, para el individuo, su felicidad y logro del pleno desarrollo pasa, necesariamente por la posibilidad de constitución y preservación de lo público. 
2) Modelos mentales. Los paradigmas manejados en organizaciones públicas y en los ciudadanos, sobre su papel en la construcción del destino de todos, deben reconstituirse y dar cuenta de la visión de mundo dentro de esa colectividad o sociedad. Los modelos mentales deben posibilitar la construcción de las conversaciones sociales y no obstaculizarlas.

3) Construcción de una visión compartida. Esta visión se construye, partiendo de la comprensión por parte de cada individuo del modo en que su visión personal puede, además de enriquecer su concepción de vida y desarrollo personales, no apartarse de la visión de la organización. De este modo, una organización debe animar a sus miembros a que creen una visión personal que de sentido a sus vidas y que posibilite el logro de la visión de la organización.

4) Aprendizaje en equipo. Las conversaciones sociales, y las sinergias cognitivas sólo pueden tener lugar en un entorno en el cual el aprendizaje sea colaborativo y cooperativo, pues sólo así es posible hacer una común construcción del sentido del bien público. El aprendizaje colaborativo y cooperativo pasa por la construcción y preservación de una memoria colectiva que cimiente el proceso del aprendizaje.

5) Pensamiento sistémico. La generación de sinergías cognitivas, el conocimiento de las capacidades y potencialidades ciudadanas y las conversaciones sociales entre organizaciones, ciudadanos y grupos de unos y otros, posibilitarán la construcción de un entramado organizativo-público que de cuenta de la complejidad de la sociedad. Es lo que pudiéramos llamar, la posibilidad de pensar a la sociedad como un proyecto, en el doble sentido de la palabra. Por una parte, muestra y "proyecta" aquello que la sociedad ha ido construyendo en su historia. Por la otra, ofrece un "horizonte de expectativas" holístico para la sociedad. Es decir, lo que es bueno y es bueno para todos.

El esbozo de estas cinco disciplinas, marcando el ritmo del aprendizaje institucional en instituciones públicas contribuirá a ilustrar la idea que se plantea en el apartado siguiente y que enfatiza en una de las características principales del aprendizaje en términos de este tipo de organizaciones que tiene que ver con su carácter colectivo.

\section{Las instituciones que aprehenden nuevos modos de construir el desarrollo endógeno}

Antes se mostró cómo el aprendizaje en los miembros de una organización se convierte en uno de sus elementos de mayor valía, pues se constituye en uno de los pilares fundamentales en el proceso de toda la organización en el camino de adquisición de conocimiento, superación de errores y adaptación al entorno, en suma, en el camino del aprendizaje.

Como se ha visto, en el contexto de la organización inteligente la visión de la organización es muy limitante y viene dada con antelación al ingreso de cada individuo a su estructura. Además, el dominio de las cinco disciplinas, como lo muestra Senge, pasa porque buena parte de la visión personal de cada uno de sus 
miembros se acople, se adapte y trabaje para la construcción de esa visión compartida que es común a todos los miembros e identifica a la organización, distinguiéndola entre sus pares, lo cual constituye el camino para la realización individual, puesto que esta visión limitante de las organizaciones no deja lugar a lo público al ser lo privado lo que forma la sociedad, constituyéndose en una suerte de secuestro de lo público desde la esfera de lo colectivo hacia la esfera de lo privado. Una vía hacia la superación de esta suerte de secuestro de lo público pasa por asumir que toda institución pública debe observar también en sus procesos y prácticas énfasis en el aprendizaje colectivo y hacia la formación ciudadana, en el entendido que el término institución pública no sólo hace referencia a una estructura organizacional o administrativa, sino también a principios socialmente aceptados como válidos y a prácticas sociales, públicas, consolidadas y aceptadas por todos los ciudadanos.

Así, se asume que las instituciones públicas son espacios en los cuales el aprendizaje del colectivo y el aprendizaje cooperativo tienen lugar. En otras palabras, el Ba al que hacen referencia Nonaka y Takeuchi (1994), en el espacio público, ocurre en un campo donde el sentido y contenido de lo público se construye partiendo de la aceptación de diversidad entre los ciudadanos, y del intercambio de sus conocimientos y apreciaciones sobre lo que el bien público debe contener y a partir de dónde debe construirse esa idea de lo público.

Esto es así, fundamentalmente, porque en el espacio de las instituciones públicas y donde se posibilita la interacción de los ciudadanos con éstas y que permite avanzar hacia la construcción del bien público, gracias al intercambio y construcción de significados. Pero para ello es fundamental partir de la premisa de la aceptación de las diferencias, asumiendo la otredad ${ }^{8}$ como un hecho y una necesidad en la construcción de ese espacio o piso común para la idea de desarrollo del ser humano y de esa colectividad.

Ahora bien, si las instituciones generan un espacio para la construcción del bien público, cabría conocer ¿en cuál espacio concurren las instituciones públicas? La respuesta a esta pregunta se ha venido esbozando a lo largo de este documento, cuando se ha mostrado la necesidad de interacción entre ciudadanos y organizaciones públicas en la constitución y mantenimiento de lo público. Pero ¿qué implica realmente esto, si se tiene en cuenta que tanto lo público como las mismas instituciones públicas tienen espacios y objetos de acción muy amplios y, por lo tanto, difusos?

Comenzaremos por definir el espacio de acción de las instituciones públicas. En primer lugar, las instituciones públicas en tanto que organizaciones encargadas de administrar lo público, anuncian su presencia en un ámbito que compete a la gestión de las relaciones entre institu- 
El desarrollo endógeno y aprendizaje institucional: Una relación recursiva Petrizzo Páez, María Angela

ciones públicas y entre los ciudadanos y éstas, en aras de cumplir con una labor que define la Administración Pública: la gestión. En este contexto, las instituciones públicas diseñan, establecen y aplican mecanismos que posibilitan la compra de insumos, pago de impuestos y ejercicio de derechos y deberes ciudadanos consagrados en las leyes, como el voto o el control del ejercicio de las instituciones públicas. En esta labor, las instituciones públicas se encargan de gestionar la correcta prestación de los servicios públicos y, para ello, en ocasiones establecen vínculos con organizaciones privadas, a las cuales se les concede, según un criterio preestablecido, la prestación de determinados servicios públicos ${ }^{9}$. Esta, si se quiere, es una revisión del modo en que puede alcanzarse la gestión de los bienes públicos como uno de los fines de la institución pública.

Finalmente, las instituciones públicas, como encargadas de custodiar el surgimiento, constitución y preservación de lo público, operan en la construcción de vínculos inter-ciudadanos, que posibiliten su aprendizaje y formación en aras de que esa tarea de constitución y preservación sea realizada entre las institucio- nes públicas y los ciudadanos. Esto sólo es posible si, además de gestionar lo público, avanzan en la formación ciudadana de los miembros de la comunidad en el marco de una labor colectiva de constitución de procesos de aprendizaje.

No se pretende aquí que la enumeración anterior sea exhaustiva. Sin embargo, permite observar que el aprendizaje es no sólo deseable, sino necesario en el marco de la interacción entre organizaciones públicas y entre éstas y los ciudadanos y que esto se hace evidente en cualquiera de los planos en que se observa la presencia de las instituciones públicas. Sin embargo, la necesidad del aprendizaje del colectivo, y del aprendizaje colectivo ${ }^{10}$, se hace aún más evidente en este último espacio enunciado, pues allí recobra su sentido último: la formación del individuo en su tránsito hacia la condición de ciudadano, y de allí en el camino hacia la construcción de procesos que desemboquen en un Desarrollo Endógeno.

De esta forma, las conversaciones sociales son un modo en el que el aprendizaje colectivo se posibilita. Pero además, las conversaciones sociales enriquecen el aprendizaje colectivo, y lo hacen también colaborativo, cooperativo y

9 Se habla aquí de los medios de comunicación, y de la prestación de servicios como recolección y tratamiento de desechos sólidos, telecomunicaciones y otros.

10 El aprendizaje del colectivo hace referencia directa a la adquisición de algunos tipos de conocimiento que sirven al Desarrollo Endógeno como el organizacional, el social, el institucional y el técnico. Sin embargo, el que el colectivo adquiera estos conocimientos, los aprenda y aprehenda, no implica necesariamente que dicho proceso sea realizado de forma colectiva. Dicho en otras palabras, aprendizaje del colectivo no necesariamente conduce a conversaciones sociales, que son lo que se produce gracias a que el aprendizaje ocurra en un espacio colectivo que posibilite la generación, intercambio y enriquecimiento de ese conocimiento $(B a)$, y en el que concurran tanto ciudadanos como instituciones públicas. 
crítico (Ochoa y Petrizzo, 2006). En primer lugar, el aprendizaje se hace colaborativo, pues el resultado es producto directo de la interacción de varios actores (ciudadanos-instituciones públicas) y no de la prevalencia de unos valores individuales sobre otros. En segundo lugar, el aprendizaje se hace cooperativo, porque permite afrontar con soluciones conjuntas situaciones que ese colectivo pueda afrontar. Finalmente es crítico, pues se espera que sea el resultado directo del contraste entre distintas formas de pensar y concebir al mundo, en lugar de ser el resultado de aquello que Senge mostraba como la disciplina del dominio personal, en donde el individuo se somete a la organización.

Ochoa y Pilonieta (2006) hablan del Desarrollo Endógeno en términos conceptuales, como resultado de un proceso de reconocimiento de la revisión del concepto desarrollo en un plano en donde se pueda plantear el ejercicio de una relación libre con la economía en la que ésta última forme parte del quehacer de la sociedad, siendo resultado de su devenir, y no estando jerárquicamente por encima de ésta, como plantea la noción clásica de desarrollo. Esto conlleva la necesidad de observar al Desarrollo Endógeno desde una perspectiva integral, en donde puedan examinarse varios modos en los que cualquier alternativa de desarrollo se despliega como garante y constructora de su endogeneidad. Cierto es que la forma de entender al Desarrollo Endógeno que se ha mostrado hasta ahora, en tanto que el puente que habilita el replanteamiento en las relaciones y vínculos entre los individuos y el Estado, dándoles a los primeros la doble condición de ciudadanos y de sujetos del desarrollo, posibilita también la definición de dimensiones o espacios en los que ese desarrollo con característica de endógeno y condición de sustentable, es reforzado. Estas dimensiones esbozadas por los autores son decisión, control y retención de beneficios y se encuentran ancladas en lo lo$\mathrm{cal}^{11}$, como referente primario. El elemento territorial, en términos de lo local, es lo que contextualiza cada una de las dimensiones del Desarrollo y el conjunto que estas forman. En el Cuadro 1 se muestra el cruce entre los elementos que componen cada una de las dimensiones y el modo en que debieran poder ser observados a la luz de cada potencialidad de las mostradas localmente.

Ahora bien, ¿qué implicaciones tiene el que, en el marco de estrategias de Desarrollo Endógeno, la decisión, control y beneficios sean garantizados localmente? Avanzar hacia la respuesta de esta pregunta, sin duda plantea su disección analítica pero, en todo caso, es fundamental entender que estas dimensiones

11 Entendiendo por local aquello que lo remite a una unidad territorial como referente inmediato pero en donde no siempre la unidad territorial de referencia es idéntica a la que se observa en una definición estrictamente geográfica del territorio. Así, la unidad territorial referente, lo local, puede ser desde una manzana, sector o parroquia, hasta un conjunto de municipios o estados circunvecinos, o incluso una entelequia compuesta por espacios geográficos cualquiera sin necesidad de ser contiguos. 
nunca concurren ni de forma lineal, ni aislada, ni mucho menos indiferente del entorno en el que ocurren. Sin embargo, la separación que se hace a continuación, es un mero recurso útil para su explicación, mas no como un referente de la realidad, en donde estas dimensiones se presentan como un entramado complejo signado por las relaciones de dependencia mutua.

En primer lugar, se dirá que la decisión sólo puede ser local si viene construida sobre la base del diálogo. Ese diálogo debe tener como naturaleza central su carácter resultante de la construcción colectiva entre ciudadanos y entre éstos y las organizaciones públicas. En este contexto, la decisión local viene garantizada por el levantamiento de la memoria colectiva de la comunidad y, por ende, de la construcción del arraigo de los ciudadanos a su entorno. Así, del diálogo local ${ }^{12}$ se desprende un conocimiento colectivo sobre vocaciones y potencialidades de los ciudadanos y de su entorno ${ }^{13}$ pero, además, se posibilita que los ciudadanos y las organizaciones avancen en la construcción de alianzas y hacia el fortalecimiento de vínculos que hagan de las materias objeto de decisión, escenarios probables. El modo en que se alcanzan las decisiones en este contexto es variado, y puede incluir tanto mecanismos formales como informales ${ }^{14}$. Sin embargo, buena parte de las decisiones incluyen, mutatis mutandis, elementos básicos tomados de la práctica de la toma de decisiones, como recolección de información, formulación de situaciones o escenarios deseables, comparación de resultados en cada escenario, evaluación de impacto y selección o decisión. El modo en que cada uno de estos elementos se muestre a lo largo de cada uno de los procesos locales de toma de decisión, podrá ser distinto en función de la materia de decisión, y de la comunidad de la que se hable. En algunos casos, estas prácticas tan asociadas a una concepción rígida de la toma de decisiones ${ }^{15}$, se encuentra tan arraigada en el subconsciente colectivo, que son utilizadas de modo intuitivo por los ciudadanos aún en estadios tempranos de organización social de los colectivos (ver Cuadro 1).

En segundo lugar, el control permanecerá en el ámbito local, en la medida en que se logre que, de las conversaciones sociales se generen las sinergías cognitivas, la construcción común de significados útiles para la empresa de construir el Desarrollo Endógeno, y se refuer-

12 Que en términos de Boisier sería conversación social.

13 Entendido como un espacio en donde ocurren relaciones de diversa índole y que sustentan la vida en sociedad.

14 Siendo los primeros los referidos a mecanismos establecidos, por ejemplo, a través de leyes que regulen la participación política, y los segundos a conversaciones informales que pueden ser considerados como germen de procesos posteriores que se construyan como una decisión.

15 Definición del problema, establecimiento de sus causas, determinación de recursos y posibles soluciones y delineación de cursos de acción futuros. 
Revista Venezolana de Gerencia, Año 13, No. 41, 2008

\begin{tabular}{|c|c|c|c|c|c|}
\hline \multicolumn{6}{|c|}{$\begin{array}{c}\text { Cuadro } 1 \\
\text { Instrumento de Observación del Desarrollo Endógeno }\end{array}$} \\
\hline & & & \multicolumn{3}{|c|}{ Unidad Territorial } \\
\hline & & & $\begin{array}{c}\text { Potencialidad } \\
1\end{array}$ & $\begin{array}{c}\text { Potencialidad } \\
2\end{array}$ & $\begin{array}{c}\text { Potencialidad } \\
3\end{array}$ \\
\hline \multirow{8}{*}{$\begin{array}{l}\text { Dimensiones } \\
\text { del Desarrollo } \\
\text { Endógeno }\end{array}$} & $\begin{array}{l}\text { Decisión } \\
\text { Local }\end{array}$ & $\begin{array}{l}\text { Espacios de } \\
\text { Diálogo }\end{array}$ & & & \\
\hline & & $\begin{array}{c}\text { Espacios de } \\
\text { organización } \\
\text { social }\end{array}$ & & & \\
\hline & & $\begin{array}{l}\text { Espacios de } \\
\text { interacción } \\
\text { ciudadana }\end{array}$ & & & \\
\hline & & $\begin{array}{l}\text { Apropiación de } \\
\text { la información }\end{array}$ & & & \\
\hline & $\begin{array}{l}\text { Control } \\
\text { Local }\end{array}$ & $\begin{array}{l}\text { Instrumentos } \\
\text { de contraloría }\end{array}$ & & & \\
\hline & & $\begin{array}{l}\text { Indicadores de } \\
\text { seguimiento }\end{array}$ & & & \\
\hline & $\begin{array}{l}\text { Retención } \\
\text { local de } \\
\text { Beneficios }\end{array}$ & $\begin{array}{l}\text { Enriquecimient } \\
\text { o sociocultural }\end{array}$ & & & \\
\hline & & $\begin{array}{l}\text { Muestras de } \\
\text { en riquecimient } \\
\text { o económico }\end{array}$ & & & \\
\hline
\end{tabular}

Fuente: Petrizzo, Aldana y Ochoa, 2006.

ce en ese espacio el proceso de aprendizaje en aras de funcionar de un modo proactivo hacia el entorno, garantizando la adaptabilidad de las decisiones a nuevos escenarios que, determinados por factores externos a la comunidad, le afecten de forma directa. Sin embargo, esta característica tan "gerencial" no es la única que tiene el control local como dimensión de observación del Desarrollo Endógeno. En el ejercicio del control sobre la decisión donde puede observarse de una manera más eficaz el despliegue de la actividad del ciudadano. Un ciudadano res- ponsable con el mantenimiento de su entorno, se comprometerá con la vigilancia del curso de las decisiones pues sabrá también que el devenir de éstas es su responsabilidad. Al igual que la dimensión de la decisión local, el proceso de control puede verse desde una óptica gerencial $^{16}$. Sin embargo, el valor enriquecedor de esta dimensión en el marco del Desarrollo Endógeno, lo constituye el hecho de que el control sobre las decisiones y su curso representa un espacio idóneo para que el ejercicio de la ciudadanía se despliegue de una forma diáfana, contribu- 
El desarrollo endógeno y aprendizaje institucional: Una relación recursiva Petrizzo Páez, María Angela

yendo a la preservación de lo que, a través de la decisión local, el colectivo ha construido de la mano con las organizaciones públicas, como bien público.

Finalmente, el enriquecimiento será local cuando se garantice que, entre otras cosas, el proceso del Desarrollo Endógeno se traduce en la generación de las sinergías cognitivas cuyos modos ilustra Boisier (2002), de forma que la relación con la economía se desarrolle de la manera más libre posible y, además, en un contexto en donde los beneficios económicos sean recontextualizados y se conviertan en uno de los indicadores del éxito más que en el indicador por excelencia de éste. El enriquecimiento local de un proceso de Desarrollo Endógeno debe ser observable en todos los contextos en que el quehacer social de ese colectivo se despliegue. Lógicamente su incidencia en el proceso de aprendizaje, en los ámbitos en los que este puede ser generado y apropiado, formal e informal, es una de las muestras más importantes de enriquecimiento local. Pero además, el enriquecimiento local debe dar cuenta de los modos en que el colectivo intenta potenciar aquellas actividades y decisiones que refuercen, en conjunto el proceso de Desarrollo Endógeno.

Ahora bien, que el Desarrollo Endógeno acaezca en una sociedad puede plantearse en función de que cada una de las dimensiones antes dichas se expresen y desplieguen al máximo posible. Pero esto último sólo es posible en la me- dida en que se pueda entender que la acción de estas dimensiones no es, en ningún caso, una acción aislada o independiente entre ellas, o con respecto al entorno en el que ocurren. Las dimensiones del Desarrollo Endógeno, en los términos en que han sido expuestas antes, se plantean en ocurrencia sinérgica e inmersas en un proceso de sinergía social que garantiza que sólo de la acción conjunta podrán operarse, de modo efectivo todos los procesos necesarios para la construcción del Desarrollo Endógeno.

\section{Conclusiones}

A lo largo de este documento se ha ilustrado la noción de aprendizaje institucional, un término tomado de su equivalente en las técnicas gerenciales el aprendizaje organizacional, aplicándolo al contexto de las instituciones públicas, e ilustrando el modo en que estos procesos constituyen el pilar fundamental del sustrato básico del Desarrollo Endógeno: las conversaciones sociales y las sinergias cognitivas.

Una vez mostrada la naturaleza del aprendizaje institucional, desde el cual se plantea la propuesta del Desarrollo Endógeno, se asume como necesario el inicio de un proceso que supone la existencia de un ámbito de aprendizaje más complejo, multidimensional y que supera lo que se entiende como organización y clima organizacional. En realidad, la propuesta de aprendizaje institucional esbozada

16 En términos de que implica recabar información, identificar medios, articularlos, ejecutar la opción escogida y hacer seguimiento de los planes 
aquí apunta hacia un proceso de aprendizaje en el que tanto las organizaciones y los ciudadanos, como las expresiones del colectivo generen un entramado social que, sustentada sobre las conversaciones sociales, pueda reconstituir el espacio de lo público más allá de lo que cada actor demande como su beneficio particular o específico, por una suerte de piso común que defina el proyecto de sociedad que se pretende construir.

En este proceso, tanto las instituciones públicas como las organizaciones más tradicionalmente vinculadas al quehacer social, emergen como espacios privilegiados para la formación ciudadana, en el entendido que esta formación debe estar alimentada por la generación de conocimiento colaborativo, cooperativo y crítico, y de que éste debe estar orientado hacia la construcción del bien público, sustentando de este modo el ejercicio de la ciudadanía a través de la construcción conjunta del Desarrollo Endógeno.

\section{Referencias bibliográficas}

Manage Rigor and Relevance (2005). SECI Model (Nonaka Takeuchi) documento disponible en internet desde http://www.12manage.com/methods _nonaka_seci_es.html consulta hecha en febrero 2007.

Argyris, C. y D. A. Schön (1978). Organizational Learning: a Theory of Action Perspective, Reading, MA, AddisonWesley.

Boisier, S. (2002). ¿Y si el Desarrollo fuera una emergencia sistémica? en Documentos de trabajo № 6 . Instituto de Desarrollo Regional Fundación Universitaria.
Brown, J. S., Collins, A. y Duguid, S. (1989). Situated cognition and the culture of learning en Educational Researcher, 18(1), 32-42.

Duncan, R.B. y Weiss, A. (1979). Organizational Learning: Implications for Organizational Design. En: Straw, B.M. y Cummings, L.L.(eds). Research in Organizational Behaviour, p. 75123. JAI Press. Greenwich CT.

Hedberg, B. (1981). How Organizations Learn and Unlearn. En: Nystrom, P y C. Starbuck, W.H., Handbook of Organizational Design, vol. 1, p.3-27. Oxford University Press. Oxford.

March, J. G. y Olsen, J. P (1976). Organizational Learning and the Ambiguity of the Past. En: March, J. G. y Olsen, J. P. (eds.), Ambiguity and Choice in Organizations, p.54-68. Universitetsforlaget. Bergen. Noruega.

McGill, M. E. y Slocum J. W. (1994). The Smarter Organization: How to Build a Business That Learns and Adapts to Marketplace Needs. Wiley. USA.

Moreno, N. y Rodríguez, F. (2002). La gestión de la información como base de la gestión del coocimiento y del aprendizaje organizacional en las universidades. En Revista Cubana de Educación Superior. Vol. XXII, no 2 de 2002. Centro de Estudios para el Perfeccionamiento de la Educación Superior, CEPES, Universidad de La Habana. pp 19-36.

Nekane A., Goya (2000). Un Estudio del Aprendizaje Organizativo desde la Perspectiva del Cambio: Implicaciones Estratégicas y Organizativas. Tesis Doctoral. Universidad de Deusto. España.

Nonaka, I. (1988). Creating Organizational Order out of Chaos: Self-Renewal in Japanese Firms, en California Management Review, Spring, p.57-93. 
El desarrollo endógeno y aprendizaje institucional: Una relación recursiva

Petrizzo Páez, María Angela

Nonaka (1991). The Knowledge-Creating Company, en Harvard Business Review, vol.69, nำ6, p.96-104.

Nonaka, I. (1994). A dynamic theory of organizational knowledge creation. En Organization Science, 5(1):14-37.

Nonaka, I. y Johansson, J.K. (1985). Japanese Management: What about the "Hard" Skills?, En Academy of Management Review, vol.10, №2, p.181-191.

Nonaka, I. y Takeuchi, H. (1995). The Knowledge-Creating Company. Oxford University Press.

Ochoa, A. y Petrizzo, M. (2006). Redes cognitivas y redes de aprendizaje. Hacia la construcción de un conocimiento crítico en Red Documento presentado en el Congreso Online de Cibersociedad, 20-11 al 03-12 del 2006. Documento disponible desde http://www.cibersociedad.net/congres2006/gts/comunicacio. php? $\mathrm{id}=634 \&$ llengua $=\mathrm{es}$

Ochoa, A. y Pilonieta, C. (2006). El Desarrollo Endógeno Sustentable. Una aproximación conceptual en Ochoa, A. (Comp.) (2006) Aprendiendo en tor- no al Desarrollo Endógeno. CDCHT - CSI - FUNDACITE Mérida.

Petrizzo, M.A. (2005). Participación ciudadana y tecnologías de la información y comunicación, Nueva Sociedad, no 195 Ene-Feb 2005, 88-101, Caracas.

Petrizzo, M. A.; Aldana, E. y Ochoa, A. (2006). Pautas para comprender al Desarrollo Endógeno en Alejandro Ochoa (ed.) Aprendiendo en torno al Desarrollo Endógeno, pp 44-50, Mérida, FUNDACITE-ULA.

Senge, P. (1990). La quinta disciplina. Cómo impulsar el aprendozake en la organización inteligente. Ediciones Juan Granica. España.

Suárez, T. (1998). La 'Quinta Disciplina' en el contexto del proyecto social de la modernidad en Revista Venezolana de Gerencia. Año 3, № 6, 1998.

Wilson, B. y Cole, P. (1991). A review of cognitive teaching models. En Educational Technology Research and Development, 39(4), 47-64. 
El desarrollo endógeno y aprendizaje institucional: Una relación recursiva

Petrizzo Páez, María Angela

Nonaka (1991). The Knowledge-Creating Company, en Harvard Business Review, vol.69, nำ6, p.96-104.

Nonaka, I. (1994). A dynamic theory of organizational knowledge creation. En Organization Science, 5(1):14-37.

Nonaka, I. y Johansson, J.K. (1985). Japanese Management: What about the "Hard" Skills?, En Academy of Management Review, vol.10, №2, p.181-191.

Nonaka, I. y Takeuchi, H. (1995). The Knowledge-Creating Company. Oxford University Press.

Ochoa, A. y Petrizzo, M. (2006). Redes cognitivas y redes de aprendizaje. Hacia la construcción de un conocimiento crítico en Red Documento presentado en el Congreso Online de Cibersociedad, 20-11 al 03-12 del 2006. Documento disponible desde http://www.cibersociedad.net/congres2006/gts/comunicacio. php? $\mathrm{id}=634 \&$ llengua $=\mathrm{es}$

Ochoa, A. y Pilonieta, C. (2006). El Desarrollo Endógeno Sustentable. Una aproximación conceptual en Ochoa, A. (Comp.) (2006) Aprendiendo en tor- no al Desarrollo Endógeno. CDCHT - CSI - FUNDACITE Mérida.

Petrizzo, M.A. (2005). Participación ciudadana y tecnologías de la información y comunicación, Nueva Sociedad, no 195 Ene-Feb 2005, 88-101, Caracas.

Petrizzo, M. A.; Aldana, E. y Ochoa, A. (2006). Pautas para comprender al Desarrollo Endógeno en Alejandro Ochoa (ed.) Aprendiendo en torno al Desarrollo Endógeno, pp 44-50, Mérida, FUNDACITE-ULA.

Senge, P. (1990). La quinta disciplina. Cómo impulsar el aprendozake en la organización inteligente. Ediciones Juan Granica. España.

Suárez, T. (1998). La 'Quinta Disciplina' en el contexto del proyecto social de la modernidad en Revista Venezolana de Gerencia. Año 3, № 6, 1998.

Wilson, B. y Cole, P. (1991). A review of cognitive teaching models. En Educational Technology Research and Development, 39(4), 47-64. 
El desarrollo endógeno y aprendizaje institucional: Una relación recursiva

Petrizzo Páez, María Angela

Nonaka (1991). The Knowledge-Creating Company, en Harvard Business Review, vol.69, nำ6, p.96-104.

Nonaka, I. (1994). A dynamic theory of organizational knowledge creation. En Organization Science, 5(1):14-37.

Nonaka, I. y Johansson, J.K. (1985). Japanese Management: What about the "Hard" Skills?, En Academy of Management Review, vol.10, №2, p.181-191.

Nonaka, I. y Takeuchi, H. (1995). The Knowledge-Creating Company. Oxford University Press.

Ochoa, A. y Petrizzo, M. (2006). Redes cognitivas y redes de aprendizaje. Hacia la construcción de un conocimiento crítico en Red Documento presentado en el Congreso Online de Cibersociedad, 20-11 al 03-12 del 2006. Documento disponible desde http://www.cibersociedad.net/congres2006/gts/comunicacio. php? $\mathrm{id}=634 \&$ llengua $=\mathrm{es}$

Ochoa, A. y Pilonieta, C. (2006). El Desarrollo Endógeno Sustentable. Una aproximación conceptual en Ochoa, A. (Comp.) (2006) Aprendiendo en tor- no al Desarrollo Endógeno. CDCHT - CSI - FUNDACITE Mérida.

Petrizzo, M.A. (2005). Participación ciudadana y tecnologías de la información y comunicación, Nueva Sociedad, no 195 Ene-Feb 2005, 88-101, Caracas.

Petrizzo, M. A.; Aldana, E. y Ochoa, A. (2006). Pautas para comprender al Desarrollo Endógeno en Alejandro Ochoa (ed.) Aprendiendo en torno al Desarrollo Endógeno, pp 44-50, Mérida, FUNDACITE-ULA.

Senge, P. (1990). La quinta disciplina. Cómo impulsar el aprendozake en la organización inteligente. Ediciones Juan Granica. España.

Suárez, T. (1998). La 'Quinta Disciplina' en el contexto del proyecto social de la modernidad en Revista Venezolana de Gerencia. Año 3, № 6, 1998.

Wilson, B. y Cole, P. (1991). A review of cognitive teaching models. En Educational Technology Research and Development, 39(4), 47-64. 
El desarrollo endógeno y aprendizaje institucional: Una relación recursiva

Petrizzo Páez, María Angela

Nonaka (1991). The Knowledge-Creating Company, en Harvard Business Review, vol.69, nำ6, p.96-104.

Nonaka, I. (1994). A dynamic theory of organizational knowledge creation. En Organization Science, 5(1):14-37.

Nonaka, I. y Johansson, J.K. (1985). Japanese Management: What about the "Hard" Skills?, En Academy of Management Review, vol.10, №2, p.181-191.

Nonaka, I. y Takeuchi, H. (1995). The Knowledge-Creating Company. Oxford University Press.

Ochoa, A. y Petrizzo, M. (2006). Redes cognitivas y redes de aprendizaje. Hacia la construcción de un conocimiento crítico en Red Documento presentado en el Congreso Online de Cibersociedad, 20-11 al 03-12 del 2006. Documento disponible desde http://www.cibersociedad.net/congres2006/gts/comunicacio. php? $\mathrm{id}=634 \&$ llengua $=\mathrm{es}$

Ochoa, A. y Pilonieta, C. (2006). El Desarrollo Endógeno Sustentable. Una aproximación conceptual en Ochoa, A. (Comp.) (2006) Aprendiendo en tor- no al Desarrollo Endógeno. CDCHT - CSI - FUNDACITE Mérida.

Petrizzo, M.A. (2005). Participación ciudadana y tecnologías de la información y comunicación, Nueva Sociedad, no 195 Ene-Feb 2005, 88-101, Caracas.

Petrizzo, M. A.; Aldana, E. y Ochoa, A. (2006). Pautas para comprender al Desarrollo Endógeno en Alejandro Ochoa (ed.) Aprendiendo en torno al Desarrollo Endógeno, pp 44-50, Mérida, FUNDACITE-ULA.

Senge, P. (1990). La quinta disciplina. Cómo impulsar el aprendozake en la organización inteligente. Ediciones Juan Granica. España.

Suárez, T. (1998). La 'Quinta Disciplina' en el contexto del proyecto social de la modernidad en Revista Venezolana de Gerencia. Año 3, № 6, 1998.

Wilson, B. y Cole, P. (1991). A review of cognitive teaching models. En Educational Technology Research and Development, 39(4), 47-64. 